\title{
LA RELACIÓN ENTRE FILOSOFÍA, CIENCIA Y RELIGIÓN: APROXIMACIÓN TEÓRICA DESDE EL ESTUDIO DE MARIANO ARTIGAS Y ALEXANDRE KOYRÉ
}

\author{
FERNANDA TUSA JUMBO, XAVIER BRICEÑO CASTILLO, \\ EDUARDO TUSA JUMBO \\ Universidad Técnica de Machala, Ecuador
}

\begin{abstract}
RESUMEN: Este artículo hace una aproximación teórica de la relación existente entre filosofía, ciencia y religión dentro del devenir de la historia de la humanidad y la cultura postmoderna. Mediante una investigación de teoría fundamentada contenida en los textos Supuestos e implicaciones del progreso científico del filósofo español Mariano Artigas, así como Pensar la ciencia y La influencia de las concepciones filosóficas en las teorías científicas del historiador ruso Alexandre Koyré, se expone la idea de que un triángulo armónico entre Dios, filosofía y ciencia no solo es compatible con el devenir de los avances científicos contemporáneos, sino que también es necesario para restablecer una etapa histórica que podríamos denominar nuevo renacimiento, vinculado, tal como dijera Artigas, a tres componentes altamente simbióticos entre sí: dimensión espiritual de la vida humana, la búsqueda objetiva de la verdad y la existencia de valores éticos al servicio de la humanidad.
\end{abstract}

PALABRAS CLAVE: Koyré; Artigas; Dios, filosofía; ciencia.

\section{The relationship between philosophy, science and religion: theoretical approach from the study of Mariano Artigas and Alexandre Koyre}

ABSTRACT:THIS article proposes a theoretical approach about the relationship between philosophy, science and religion in the evolution of human history and postmodern culture. Using a grounded research theory by mean of the analysis of few texts such as: Assumptions and implications of scientific progress written by Mariano Artigas, spanish philosopher, and the texts: Thinking Science and The influence of philosophical concepts in scientific theories written by Alexandre Koyre, russian historian, this article explores the following thesis: a harmonic triangle among God, philosophy and science not only supports the evolution of contemporary scientific advances but also is necessary to restore a historic period might be called new renaissance, linked, as said Artigas, influenced by three highly symbiotic components: spiritual dimension of human life, objective search for truth and the existence of ethical values, which help to citizenships to development a good humanity service.

KEY WORDS: Koyré; Artigas; God; philosophy; science.

INTRODUCCIÓN

Dios es la evidencia invisible Víctor Hugo

Nunca antes habían sido tan criticados conceptos como fe, dogmatismo y religión, dentro de una humanidad vista a sí misma como altamente racional, escéptica y objetiva. Dentro de la coyuntura social a nivel de la globalización, fenómenos como los atentados de Charlie Hebdo, el terrorismo, las guerras, el fanatismo religioso, la pobreza, la crisis del medio ambiente, la ética de investigaciones en laboratorios 
y otros tantos sucesos han aumentado el abismo de cuestionamientos y rechazos entre ciencia, filosofía y religión.

Pocos son los colectivos sociales, a día de hoy, que conservan una auténtica fe viva por un creador omnipresente, principio y fin de todas las cosas. Y digo auténtica, porque según los datos del Anuario Estadístico del Vaticano solamente en el año 2012 había 1229 millones de católicos en el mundo, de los cuales el 49\% se encuentra en el continente americano; sin embargo, y esto será materia de una nueva investigación, habría que determinar quienes practican a consciencia una devoción y compromiso de fe con sus principios religiosos, o al contrario, manejan una teología a medias; y aún más, sería interesante conocer cuántos científicos creyentes y no creyentes existen en el mundo.

El efecto dominó de este panorama es que el debate científico actual busca prescindir de la figura de Dios dentro de sus concepciones filosóficas en miras a restaurar, lo que a su criterio debería ser, una ciencia objetiva, racional y autosuficiente en sí misma, que constituya la nueva visión del mundo y la cultura contemporánea, pues para la comunidad de físicos, investigadores, biólogos, etc, la incorporación de Dios, dentro del panorama de avances y progresos en materia experimental, es incompatible con el carácter autónomo de este saber, considerando a la doctrina teológica como un conjunto de especulaciones teóricas y metafísicas que empañan la naturaleza intrínseca de la ciencia.

En este sentido, el paradigma evolucionista del origen de las especies del naturalista inglés Charles Darwin y la tesis de que el universo es su propio creador, contenida en Breve historia del tiempo del físico teórico Stephen Hawking se erigen como la nueva bandera del pensamiento científico, tomando a Dios, en palabras del físico francés Pierre Simon Laplace, como «una hipótesis ya no necesaria de sostener» (Laplace, 1895). Visión adoptada dentro de la comunidad científica a manera de quiebre frente al paradigma judeo-cristiano, el cual sostiene la existencia de un Dios creador, omnipresente, principio y fin de todas las cosas.

En este escenario encontramos una sociedad llena de más preguntas que certezas, desorientada, absorta en sus dudas, vulnerable hacia las tendencias ideológica-políticas que se tratan de imponer desde los estados, individuos sin horizontes ni referentes, perdidos en la semiótica del significado sin significante, del pseudo valor ético, del principio moral a medias, del ¿para qué? mantener la integridad humana. $\mathrm{Y}$ es que el imaginario social se ha establecido, en palabras del poeta argentino Oliverio Girondo, como «creo que creo en lo que creo que no creo y creo que no creo en lo que creo que creo» (Girondo, 1932) configuración de una cultura contemporánea sin luces, perdida entre la divulgación de ciencia de una educación positivista heredada, la cual conlleva la noción ulterior de quiebres y partes independientes entre ciencia, filosofía y religión.

Y muchos de nosotros, sin lugar a dudas, hemos crecido en esa ambigüedad de enseñanza-aprendizaje desvinculada entre ciencia por un extremo y filosofía y religión por el otro. Pensar en una coexistencia de estos tres elementos era un absurdo para quien se vea a sí mismo como libre pensador y divulgador de la ciencia. Pero entonces, encontramos autores como Mariano Artigas y Alexandre Koyré que nos hablan justamente de lo contrario. Y el análisis bibliográfico y de contenido de estos dos autores en torno a la relación entre filosofía, ciencia y religión será nuestro objeto de estudio dentro del presente artículo. 


\section{Metodología}

Se aplica la investigación cualitativa de teoría fundamentada y estudio de caso con aproximación teórica. Para ello se aplica:

- Análisis cualitativo de contenido.

- Revisión bibliográfica y documental (análisis de documentos, fichas bibliográficas. documentación oficial relacionada a la temática central).

\section{Desarkollo conceptual}

En su obra Dios y espacio, espíritu y materia acerca de los estudios del filósofo inglés Henry More sobre la divinidad del espacio, Alexandre Koyré escribe: «la infinitud extraña necesidad. El espacio no sólo es real, sino que es algo divino, la entidad infinita y extensa que lo invade y abarca todo es espíritu, no un espíritu, sino el espíritu, esto es Dios» (Koyré, 1979).

Bajo esta perspectiva, Dios, como diría Santo Tomás de Aquino, tiene un poder activo infinito sobre las cosas, convirtiéndose en envolvente, sin confundirse ontológicamente con el universo creado. Aquí cabría hacernos la pregunta ¿cómo es posible que la noción de Dios encaje perfectamente en el inicio del big bang, en el instante de la gran explosión que originó lo que hoy llamamos vida? Al respecto, Henry More dice: «esta entidad infinita, inmóvil y extensa aparecerá no solo como algo real, sino también como algo divino. Esto nos asegurará aún más de que no puede ser una nada» (Koyré, 1979).

Dios forma parte del génesis de la historia del tiempo al obedecer a los muchos títulos con que los metafísicos atribuían su noción del ser creador, títulos descritos como:

«(...) el primer ser es uno, simple, inmóvil, eterno, completo, independiente, existente en sí mismo, subsistente por sí mismo, incorruptible, necesario, inmenso, increado, incircunscrito, incomprensible, omnipresente, incorpóreo, omnipenetrante, omniabarcante, ser por su esencia, ser actual, acto puro» (Koyré, 1979).

Henry More lo expresa así: «un mundo indeterminadamente vasto, aunque finito, inmerso en un espacio infinito es la única concepción que nos permite mantener la distinción entre el mundo creado contingente y el Dios eterno existente a ser y per se» (Koyré, 1979).

Dios es un paradigma, un mito que explica el mundo, que le da sentido, es una filosofía que determina nuestro horizonte científico. Alexandre Koyré lo sintetiza en estas palabras: «la influencia de las concepciones filosóficas sobre el desarrollo de la ciencia ha sido tan grande como el de las concepciones científicas en el desarrollo de la filosofía» (Koyré, 1979).

En consecuencia, Koyré (1979) manifiesta que es casi normal hablar de la influencia del pensamiento científico en la evolución de las concepciones filosóficas, no obstante se dice mucho menos de la influencia de la filosofía en la evolución del pensamiento científico, y se menciona esta influencia solo para mostrarnos que la filosofía había dominado y esterilizado la ciencia antigua y medieval en contraste con la revolución científica del siglo XVII que, a través de una liberación progresiva, logró emanciparse de la tiranía filosófica. 
También vale la pena mencionar que ciertos historiadores pensaron que los prefacios y las introducciones filosóficas, convicciones sinceras y profundas de los grandes creadores de la ciencia moderna, no eran más que gestos corteses de poca importancia en relación al trabajo real, sentado en las bases firmes de la experiencia.

Así, la filosofía se presenta como la metáfora de un andamio que ayuda al investigador a formar y a formular sus concepciones científicas y que, una vez acabada la construcción teórica, podía ser eliminada, pues para la ciencia lo que en verdad cuenta es el descubrimiento efectivo, la ley establecida. Sin embargo, y cito a Koyré, «la necesidad absoluta de estos andamios sostiene la construcción y la hace posible, el pensamiento científico puede rechazarlo post factum, pero sólo para reemplazarlos con otros» (Koyré, 1979).

Es así que la filosofía es un algo inherente al desarrollo de la ciencia. Koyré nos habla de tres enseñanzas de la historia del pensamiento científico en torno a las subestructuras o fundamentos metafísicos:

1) El pensamiento científico nunca ha estado enteramente separado del pensamiento filosófico.

2) Las grandes revoluciones científicas siempre han sido determinadas por cosmovisiones o cambios de concepciones filosóficas.

3) El pensamiento científico siempre se encuentra en el interior de un cuadro de ideas, de principios fundamentales, de evidencias axiomáticas, consideradas como pertenecientes a la filosofía.

Con estas conjeturas, Koyré expresa que la presencia de un ambiente y de un marco filosófico es condición indispensable de la existencia misma de la ciencia, aludiendo la idea del impulso creador del pensamiento. Es importante mencionar dos ejemplos del autor que muestran en sí mismos la relación entre filosofía y ciencia.

Por un lado nos habla de las concepciones cosmológicas y físicas de Aristóteles rechazadas en la ciencia moderna frente a las ideas newtonianas vistas como verdaderas, evidentes y naturales. Koyré declara lo siguiente:

«(...) la concepción aristotélica es una concepción metafísica, su cosmología tenía que ver con concepciones metafísicas muy difíciles y aquellos que no tenían una formación filosófica suficiente no podían elevarse por encima de la imaginación geométrica, no podían comprender la ciencia. Para Aristóteles, la percepción y la especulación matemática, la experiencia y no el razonamiento geométrico a priori, es lo que formaba para él el fundamento de la ciencia verdadera de lo real» (Koyré, 1979).

Por su parte, Koyré retrata el caso de Newton de forma diferente. Para el autor,

«(...) cuando tratan de explicarnos la estructura del mundo newtoniano nos dicen que ahí hay concepciones matemática bastante difíciles que aquellos que carezcan de formación matemática necesaria serán incapaces de comprenderlas como es preciso, dándose una tentativa de construir el mundo del espacio puro plena y enteramente geometrizado, sustituyéndose el mundo cualitativo del sentido común y la vida cotidiana por el mundo de la geometría reificada» (Koyré, 1979).

Desde la postura crítica de Koyré, la tesis de las experiencias alegadas por los promotores de la ciencia no prueba certezas, son solo hipótesis, ya que la historia 
del pensamiento científico es un «cementerio de errores o colección de monstruos, relegados al gabinete trastero, solamente para una obra de demolición. La lección de la historia nos enseña a ser prudentes» (Koyré, 1979).

Los programas de investigación se van sucediendo, tal cual el renacer de preocupaciones espirituales y preocupaciones cosmológicas que no son más que cambios en las formas de pensar. Al pensar se ve otra cosa, produciéndose una especie de radio de visión dentro del cual las hipótesis intentan una aproximación de la verdad y el orden del mundo dado.

Alexandre Koyré concluye diciéndonos:

«(...) el nacimiento de la ciencia moderna es concomitante de una transformaciónmutación de la actitud filosófica, de una inversión del valor atribuido al conocimiento intelectual comparado con la experiencia sensible, del descubrimiento del carácter positivo de la noción de infinito. De ahí que sea totalmente pertinente que la infinitización del universo fuera obra de un filósofo» (Koyré, 1979).

Podríamos asociar esta idea con el criterio del cardenal renacentista, Maffeo Barberinni, quien expone la noción de contingentismo epistemológico cuando afirma que Dios ha creado un mundo ordenado pero la mente humana no podrá saber de qué modo lo ha hecho, contingencia que se detiene frente a los límites de la inteligencia humana.

Barberinni nos habla de adivinanzas que no terminan nunca, hipótesis abiertas que tratan de suponer el orden del mundo cosmológico, juegos mentalesmatemáticos que intentan acercarse al conocimiento de la mente de Dios. De ninguna manera sabremos la verdad de sus reflexiones; pero vamos viendo que el resultado de nuestra profunda imaginación continúa transformándose dentro de la entropía del espacio-tiempo.

En el mismo camino, se nos presenta la lectura Supuestos e implicaciones del progreso científico de Mariano Artigas, autor que expone la configuración de una cultura contemporánea basada en métodos y resultados de la ciencia experimental, donde se prescinden de la acción divina al considerarla imposible e incluso inútil a la luz del progreso científico. Sin embargo, sostiene que es plenamente coherente la afirmación de un Dios personal creador con el reconocimiento de las dimensiones espirituales de la persona humana y con la existencia de valores éticos relacionados con la búsqueda objetiva de la verdad y el servicio a la humanidad.

En el capítulo VI del artículo antes mencionado, Artigas aborda el problema de la explicación última del universo, manifestando que la cosmovisión actual muestra al mundo atravesado por una especie de inteligencia inconsciente, cuyo nivel constitutivo refleja cierto dinamismo, inteligencia sofisticada, un complejo proceso de integración de sistemas que resulta en un elevado grado de organización, direccionalidad y cooperatividad.

Para el autor, dentro de la fábrica del universo, no hay dificultad de combinar la omnisciencia y la omnipotencia divina con la existencia de factores causales en el acontecer natural. "Somos el resultado de fuerzas ciegas que se despliegan mediante la combinación del azar y la necesidad, puede existir un plan y éste comenzó con la gran explosión o big bang» (Artigas, 1999).

Dentro de la cosmovisión científica actual podríamos afirmar, insiste Artigas, en que el mundo no ha existido siempre tal y como lo conocemos, ya que éste es resultado 
de una evolución de estados anteriores que incluye, por qué no decirlo, una multitud de novedades ontológicas especialmente significativas. En este sentido, parecería lógico admitir que Dios no ha querido crear de una sola vez todo lo que existe, sino que ha preferido crear el universo en un estado incompleto, con la capacidad de desplegar unas virtualidades cuya actualización conduce a nuevos estados.

Según su postura, «esta representación implica que el plan creador parece extenderse a lo largo de enromes períodos de tiempo, la creatividad de la naturaleza iría de la mano con la acción divina que la hace posible. Dios desea contar habitualmente con la acción de las criaturas de acuerdo con las virtualidades que Él mismo les ha otorgado» (Artigas, 1999).

Bajo esta mirada, Artigas cita un pensamiento de Santo Tomás de Aquino donde relaciona la acción de Dios con la metáfora de los leños. Al respecto, escribe

«(...) la naturaleza no es otra cosa que el plan de un cierto arte (a saber, el arte divino), impreso en las cosas, por el cual las cosas mismas se mueven hacia un fin determinado: como si el artífice que fabrica una nave pudiera otorgar a los leños que se moviesen por sí mismos para formar la estructura de la nave» (Artigas, 1999).

En consecuencia, para descubrir a Dios debemos inscribirnos dentro del desarrollo ordinario de la actividad natural; en este sentido entra en escena el progreso científico, conocimiento que detalla cada vez más la naturaleza y sus caminos. Desde dicha perspectiva, «la creatividad de la naturaleza implica elevados grados de racionalidad y organización que se comprenden a la luz de la acción divina que abraca continuamente todo lo creado. La actividad científica manifiesta de modo palpable la singularidad humana» (Artigas, 1999).

Este filósofo español establece que la hegemonía del naturalismo en el mundo está cada vez más asentada. La presunta auto-creación del universo, la autonomía propia de las ciencias naturales, el control experimental, la mentalidad positivista, la importancia del razonamiento, la búsqueda de explicaciones y la interpretación de una creación sin creador. En palabras de Artigas,

«(...) no deja lugar para las explicaciones espirituales y sobrenaturales, como si Dios no existiera, negando su posible acción en el mundo. Se ha pasado a una visión monolítica en la que parece suficiente contar con la naturaleza: al fin y al cabo, la ciencia parece permitirnos prescindir de Dios, y esa misma ciencia, nos da explicaciones de lo humano en clave materialista, lo que podría explicar una auto-producción del universo inicial sin necesidad de postular un Creador» (Artigas, 1999).

La conciliación entre ciencia, filosofía y religión para Artigas está centrado justamente en los supuestos e implicaciones de la ciencia que son en sí mismos condición necesaria para que ésta sea posible y tenga sentido. Artigas señala que «las ciencias se apoyan sobre unos supuestos filosóficos y el progreso científico retro-actúa sobre esos supuestos: los retro-justifica, los amplía y los precisa» (Artigas, 1999).

Artigas clasifica los supuestos generales de las ciencias en ontológicos, epistemológicos y antropológicos, los cuales expresan dimensiones de la naturaleza y de la persona humana sin cuya existencia la actividad científica no se daría puesto que «la reflexión filosófica puede proporcionar el complemento que la ciencia natural 
necesita para que sus resultados sean integrados en una cosmovisión unitaria que incluya diferentes dimensiones de la experiencia humana» (Artigas, 1999).

Dentro de los supuestos ontológicos de la actividad científica se destaca la racionalidad de su naturaleza misma, su grado elevado de organización estable, su orden contingente que va al mismo ritmo que el progreso de la ciencia y cuyo sinfín de procesos demuestra que la naturaleza es tan creativa como la creatividad humana. Artigas lo condensa de este modo: «la racionalidad de la naturaleza es un supuesto ontológico básico, los científicos lo admiten desde el mismo momento en que empiezan a trabajar como científicos» (Artigas, 1999).

En cuanto a los supuestos epistemológicos de la ciencia experimental, estos contienen una peculiar combinación de sensibilidad y racionalidad, cualidades inherentes a la persona humana. Bajo su perspectiva, «la actividad científica persigue ese doble objetivo, teórico y práctico ...la teoría tiene que estar construida de modo que sea posible idear experimentos que la sometan a prueba y la experimentación sólo puede realizarse si tenemos un plan racional para realizarla e interpretar sus resultados» (Artigas, 1999).

La filosofía nuevamente entra escena y Artigas añade a este supuesto el componente de creatividad científica, ciencia experimental que se caracteriza por el dinamismo cambiante de sus hipótesis, las cuales se encuentran en estrecha relación con las posibilidades del conocimiento humano, basadas no solo en datos disponibles sino en nuevos criterios para interpretar los resultados de los experimentos, lo que exige construcciones teóricas audaces. A criterio del autor, «los genios fueron capaces de aventurarse en una empresa que era exploradora y descubridora, eminentemente creativa ...ya los descubrimientos científicos sólo son posibles gracias a una dosis grande de creatividad teórica y experimental» (Artigas, 1999).

De igual manera, Artigas no deja de mencionar los supuestos antropológicos de la ciencia dentro de los cuales la verdad del conocimiento desempeña un importante papel en la existencia del hombre, ya que gracias a los enormes avances en materia de la naturaleza se ha transformado completamente las condiciones de vida de la humanidad.

En otras palabras, la actividad del individuo cambia en concordancia con los paradigmas y tesis científicas, así como con la evolución de las aplicaciones tecnológicas. Actividad científica que contribuye al fomento de valores éticos, expresados, en palabras del físico y filósofo argentino Mario Bunge, de la siguiente manera:

«la adopción universal de una actitud científica puede hacernos más sabios, nos haría más cautos en la recepción de información, en la admisión de creencias y en la formulación de previsiones, nos haría más exigentes en la contrastación de nuestras opiniones y más tolerantes con otros, nos haría más dispuestos a inquirir libremente acerca de nuestras posibilidades, y a eliminar mitos consagrados, robustecería nuestra confianza en la experiencia, guiada por la razón, y nuestra confianza en la razón contrastada por la experiencia; nos estimularía a planear y controlar mejor la acción, a seleccionar nuestros fines y a buscar normas de conducta con el conocimiento disponible, daría más vida al amor a la verdad, a la disposición a reconocer el propio error, a buscar la perfección y a comprender la imperfección inevitable, nos daría una visión del mundo eternamente joven, basadas en teorías contrastadas y nos animaría a sostener una visión realista de la vida humana, una visión equilibrada, ni optimista ni pesimista» (Artigas, 1999). 
Dentro de los valores inherentes de la actividad científica, Artigas hace énfasis en el sentido de responsabilidad, vinculado al concepto de sensibilidad. En cuanto a los valores propios del ser científico, el autor manifiesta que el amor a la verdad lleva a actuar limpiamente dentro del trabajo experimental, con escrupuloso rigor en cuanto a la divulgación de resultados, siendo conscientes de su autoridad, tratando en lo posible de cultivar una actitud de rigor y modestia intelectual. Dichas actitudes conducen a posiciones de apertura y colaboración con otras disciplinas del saber humano.

El libre albedrío de Dios es una constante en el discurso de Artigas, el cual concluye con la idea de los futuros retos creativos y los problemas morales que nos sitúan en el nivel propio de la persona dentro del campo de la ciencia. Al respecto escribe: "tomar consciencia de nuestra capacidad creativa conduce a una mayor responsabilidad ética. Dios cuenta con nosotros, con nuestra libertad, con nuestra responsabilidad, con nuestra creatividad, para realizar sus planes» (Artigas, 1999).

\section{Conclusiones}

La física y la historia de la física son tan importantes como la filosofía o la historia de la filosofía. Ambas son complementarias puesto que no puede haber teoría sin un encuentro de horizontes entre paradigmas y estructuras de pensamiento. Las ciencias naturales y exactas requieren un estudio interdisciplinario e intersubjetivos con otros campos de conocimiento humano. El problema de nuestra herencia educativa positivista es que hemos aislado la teoría de la enseñanza de la ciencia. Al estudiar física no debemos pensar solo en física, sino en autores, en torno a ir involucrándonos en todo el contexto para entender el texto, analizando el que a partir del por qué.

Bajo esta perspectiva, el diálogo entre fe y ciencia obedece a un orden epistemológico ligado a una historicidad que antecede el debate actual. Si juzgamos el origen de la vida desde el testeo empírico, interpretando el sistema de las apariencias sensibles como la única referencia posible, no estamos admitiendo más que una sola solución, estableciendo, en consecuencia, una hipótesis falsada por los datos.

Dentro del estudio del filósofo alemán Hans-Georg Gadamer (1991) él nos dice:

«(...) todo mensaje se escribe y se interpreta dentro de un horizonte de precomprensión». En física y matemática hay horizontes de pre-comprensión; sin embargo, existe la concepción errónea que el científico no hace hermenéutica, no hace interpretación, ellas ven hechos y punto. No obstante, los libros de ciencia vienen de un horizonte religioso-mítico, de ideas que son una constante dentro de la investigación, hablar de no tener filosofía va de un defecto del conocimiento a una imposibilidad real» (Gadamer, 1991).

El riquísimo despliegue de la creatividad humana obliga al investigador a buscar permanentemente contextos pragmáticos, horizontes, construcciones sociales. En este sentido, los estudios de Darwin y Hawking son teorías no contradichas hasta el momento, sin embargo, la hipótesis nunca deja de ser hipótesis.

De estos dos tratados teóricos de Alexandre Koyré en su estudio de Henry More, y la lectura de Mariano Artigas se puede concluir que: 
«(...) el progreso científico actual es plenamente coherente con un Dios que es trascendente, distinto del universo, pero a la vez inmanente, presente en todo el universo y en cada una de sus partes, dándoles continuamente el ser y todas sus virtualidades y haciendo posible el despliegue de esas virtualidades. Esta perspectiva ayuda a comprender que la creencia en Dios favorece la responsabilidad y la creatividad que va de la mano con el futuro ético de la humanidad» (Artigas, 1999).

Recordando el aporte descrito con anterioridad sobre Santo Tomás de Aquino acerca de la nave y los leños, se demuestra la tendencia hacia la auto-organización y autorrealización del ser humano. La creatividad humana se constituye así como un gen libertario dispuesta en nosotros por un orden que va más allá de nuestra comprensión y que a día de hoy, no podemos concebir qué es, hay algo más allá del cerebro. Al respecto Stephen Hawking dice: «el universo no solo tiene una historia, sino cualquier historia posible...si algún día comprendiéramos todas las leyes del universo, llegaríamos a conocer la mente de Dios» (Hawking, 1988).

Las concepciones filosóficas de los científicos deberían dotar de flexibilidad paradigmática su noción de Dios dentro del panorama evolucionista-histórico del universo ya que no se ha demostrado que exista una causalidad certeza del origen mismo de la vida, dando el margen a compatibilizar las bases teológicas con el evolucionismo de la creación, nuevamente todo es una conjetura en un universo indeterminista de causas aparentes.

A manera de conclusión se presenta el cuento El Milagro Secreto del escritor argentino Jorge Luis Borges, quien narra la historia de Jaromir Hladík, autor de la inconclusa tragedia Los enemigos. Un día antes de ser ejecutado en prisión, Hladík sostiene una oración con Dios: «si de algún modo existo, si no soy una de tus repeticiones y erratas, existo como autor de Los enemigos. Para llevar a término ese drama, que puede justificarme y justificarte, requiero un año más. Otórgame esos días, Tú de Quien son los siglos y el tiempo»(Borges, 2002). Dios le concede el milagro de detener el tiempo para que el escritor pueda justificar su existencia a partir de la creación de una pieza magistral literaria.

Se toma a rememoración este cuento porque si Dios es el creador de todas las cosas y nosotros somos su creación por excelencia podemos concluir que al preguntarnos día a día quiénes somos, de dónde venimos y hacia dónde vamos dejamos por sentado que el libre albedrío del pensamiento no es una casualidad de la evolución, sino un arte del reino espiritual, del reino físico, donde todas las conjeturas se dan respuesta entre sí y que si supiéramos responder conoceríamos finalmente la justificación de nuestra existencia.

Si buscamos a Dios, corremos el riesgo de quedarnos ciegos. Por ello, tener la certeza de ese algo tangible, empírico y demostrable está vedado al intelectualismo y la razón, salvo dentro de la ciencia, porque la ciencia es el camino de la fe, principio de todas nuestras reflexiones.

\section{BiBLIOGRAFÍA}

Artigas, M. (1999). La mente del universo. Pamplona: Enusa.

Artigas, M. (1998). Lógica y ética en Karl Popper. Pamplona: Eunsa.

Artigas, M. (1999). Filosofía de la ciencia. Pamplona: Eunsa, 291. 
Artigas, M. (1992). La inteligibilidad de la naturaleza. Pamplona: Eunsa, 1992, pp. 540.

Artigas, M. (1989). Filosofía de la ciencia experimental. Pamplona: Eunsa, pp. 419171

Artigas, M. (1985). Ciencia, razón y fe. Madrid: Libros MC, pp. 187.

Artigas, M. (1992). El hombre a la luz de la ciencia. Madrid: Madrid, pp. 254.

Artigas, M. (1998). Supuestos e implicaciones del progreso científico, en Scripta Theologica, pp. 205-225.

Artigas, M. (1998). Filosofía de la naturaleza. Pamplona: Eunsa, pp. 331.

Artigas, M, y Shea, W. (2006). W.: Galileo observed. Chicago: Walton Publishing.

Borges, J. (2002). El Milagro Secreto-Ficciones. Madrid: Alianza Editorial.

Gadamer, H. (1991). Verdad y Método. Salamanca: Sígueme.

Girondo. O. (1932). Espantapájaros. Buenos Aires: Losada.

Hawking, S. (1988). A brief history of time. New York: Bantam Books.

Koyré, A. (1988). Estudios de historia del pensamiento científico. Madrid: Siglo XXI Editores. Koyré, A. (1994). Pensar la ciencia. Barcelona: Paidós.

Koyré, A. (1979). Del mundo cerrado al mundo infinito. Madrid: Siglo XXI.

Koyré, A. (1980). Estudios galileanos. Madrid: Siglo XXI.

Laplace, P. (1895). Philosophical essay on probabilities. Fifth French Edition. New York: Spinger.

Popper, K. (1983). Conjetura y refutaciones. Barcelona: Paidós.

Zanotti, G. (1997). Investigación científica y pensamiento prudencial. Roma: Acta Philosophica

Zanotti, G. (2002). Feyerabend en serio. Madrid: Studium.

Zanotti, G. (2005). Hacia una hermenéutica realista. Buenos Aires: Universidad Austral.

Zanotti, G. (2005b). Feyerabend y la dialéctica del Iluminismo. Madrid: Studium.

Zanotti, G. (2009). «Thomas Kuhn: el paso de la racionalidad algorítmica a la racionalidad hermenéutica», en: Revista de Análisis Institucional, 1-55.

Universidad Técnica de Machala, Ecuador

ftusa@utmachala.edu.ec

xaviergeovanny.ps@gmail.com

etusa@utmachala.edu.ec

Fernanda Tusa Jumbo, Xavier Briceño Castillo, Eduardo Tusa Jumbo

[Artículo aprobado para publicación en diciembre de 2016] 